\title{
Utilization of Symmetric Switching Functions in the Symbolic Reliability Analysis of Multi-State $\boldsymbol{k}$-out-of- $\boldsymbol{n}$ Systems
}

\author{
Ali Muhammad Ali Rushdi \\ Department of Electrical and Computer Engineering \\ Faculty of Engineering, King Abdulaziz University \\ P. O. Box 80204, Jeddah, 21589, Saudi Arabia \\ E-mail-arushdi@kau.edu.sa
}

(Received January 1, 2019; Accepted January 24, 2019)

\begin{abstract}
Symmetric switching functions (SSFs) play a prominent role in the reliability analysis of a binary $k$-out-of- $n$ : $G$ system, which is a dichotomous system that is successful if and only if at least $\mathrm{k}$ out of its $\mathrm{n}$ components are successful. The aim of this paper is to extend the utility of $S S F s$ to the reliability analysis of a multi-state $k$-out-of- $n$ : $G$ system, which is a multi-state system whose multi-valued success is greater than or equal to a certain value $j$ (lying between $l$ (the lowest output level) and $M$ (the highest output level)) whenever at least $k_{m}$ components are in state $m$ or above for all $m$ such that $l \leq m \leq j$. This paper is devoted to the analysis of non-repairable multi-state $k$-out-of- $n$ : $G$ systems with independent non-identical components. The paper utilizes algebraic techniques of multiple-valued logic (together with known properties of $S S F s$ ) to evaluate each of the multiple levels of the system output as an individual binary or propositional function of the system multi-valued inputs. The formula of each of these levels is then written as a probability-ready expression, thereby allowing its immediate conversion, on a one-to-one basis, into a probability or expected value. The symbolic reliability analysis of a commodity-supply system (which serves as a standard gold example of a multi-state $k$-out-of- $n$ : $G$ system) is completed successfully herein, yielding results that have been checked symbolically, and also were shown to agree numerically with those obtained earlier.
\end{abstract}

Keywords- System reliability, Probability-ready expression, $k$-out-of- $n$ system, Multi-state system, Multiple-valued logic, Boolean quotient, Checking symbolic reliability, Variable-entered Karnaugh map.

\section{Introduction}

The reliability literature deals mainly with binary or dichotomous systems, in which both a system and its components have two states (i.e., either operational (successful) or non-operational (failed)). However, in many practical situations, there are multiple levels of system capacity or performance and/or different component performance levels and multiple component failure modes having different impacts on the system performance. These systems are modeled as multistate systems (MSSs), which might be coherent or non-coherent. This paper deals with a prominent class of coherent MSSs, viz., non-repairable $k$-out-of- $n$ MSSs with independent nonidentical components. The main contribution of the paper is to demonstrate that, similarly to binary $k$-out-of- $n$ systems, multi-state $k$-out-of- $n$ systems can be conveniently analyzed with the aid of symmetric switching functions ( $S S F s)$.

The literature abounds with many efficient techniques for the reliability analysis of MSSs (Veeraraghavan and Trivedi, 1994; Levitin et al., 2003; Zang et al., 2003; Muselli, 2005; Shrestha et al., 2007; 2010; Amari et al., 2010; Li et al., 2011; Li and Kapur, 2011; Ram, 2013; Ren et al., 2018), with many of these techniques devoted to $k$-out-of- $n$ MSSs (Zuo et al., 2007; Tian et al., 2008; Zhao and Cui, 2010; Ding et al., 2012; Levitin, 2013; Singh and Ram, 2014; Li et al., 2014; Khorshidi et al., 2015; Mo et al., 2015; Song et al., 2017). The paper utilizes algebraic techniques of multiple-valued logic (together with known properties of $S S F S$ ) to evaluate each of the multiple 
International Journal of Mathematical, Engineering and Management Sciences

Vol. 4, No. 2, 306-326, 2019

https://dx.doi.org/10.33889/IJMEMS.2019.4.2-025

levels of the system output as an individual binary or propositional function of the system multivalued inputs. The formula of each of these levels is then written as a probability-ready expression (see Appendix A), thereby allowing its immediate conversion, on a one-to-one basis, into a probability or expected value. The paper strives to provide a pedagogically-insightful treatment that establishes a clear and interesting interrelationship between binary modeling and MSS modeling by stressing that multi-valued concepts are natural and simple extensions of twovalued ones. The paper makes its point through the multi-valued analysis of a specific (albeit standard) multi-state commodity-supply system. Our results have been checked symbolically with the aid of variable-entered Karnaugh maps. Moreover, these results provide a truly independent means to check and verify earlier (and future) numerical solutions of this problem. The present analysis can be extended to other MSSs of comparable sizes, and might be automated to handle more general MSSs that are of larger sizes.

The organization of the remainder of this paper is as follows. Section 2 surveys important properties of symmetric switching functions $(S S F s)$, and hence paves the way for Section 3 to review a prominent class of these functions, namely monotonically non-decreasing ones, which represent successes of binary $k$-out-of- $n$ : $G$ systems. Section 4 provides an ample verbal and multi-valued logical description for a commodity-supply system, a standard $k$-out-of- $n$ multi-state system that is solved throughout this paper. Section 5 presents the main contribution of this paper, as it details the multi-valued logical analysis of the aforementioned system. This analysis adapts several important concepts of switching algebra to multi-valued logic, including those of probability-ready expressions, Boolean quotients, and the Boole-Shannon expansion. Section 6 discusses methods for hand-checking the resulting MSS symbolic reliability expressions, and uses variable-entered Karnaugh maps to verify the analysis in the case of non-identical components. Section 7 shows that our numerical results exactly agree with those obtained by Tian et al. (2008) and later by Mo et al. (2015). Section 8 concludes the paper. Three appendices are included to make the paper self-contained. These appendices add a multi-valued flavor to concepts that are well-known in the two-valued logical analysis of reliability systems. Appendix A provides a quick overview of probability-ready expressions, while Appendix B reviews the concept and mathematics of Boolean quotients. Appendix $\mathrm{C}$ briefly describes the Boole-Shannon expansion and its utility in the recursive description of the successes of $k$-out-of- $n$ : G MSSs.

\section{Properties of Symmetric Switching Functions}

This section reviews some of the essential properties of symmetric switching function (SSFs). More and detailed information about SSFs in general is available in Caldwell (1954), Marcus (1956), Arnold and Harrison (1963), Cunkle (1963), Born and Scidmore (1968), Lee (1978), Muroga (1979), Unger (1989), Kim and Dietmeyer (1991), Hill and Peterson (1993), Kravets and Sakallah (2000), Mishchenko (2003), Canteaut and Videau (2005), and Maurer (2015).

Specific exposition of the utility of SSFs in the reliability analysis of binary $k$-out-of- $n$ systems is given in Rushdi (1986, 1993).

A symmetric switching function $(S S F)$ is defined as

$f=\operatorname{Sy}(n ; \boldsymbol{A} ; \boldsymbol{X})=\operatorname{Sy}\left(n ;\left\{a_{1}, a_{2}, \ldots, a_{m}\right\} ; X_{1}, X_{2}, \ldots, X_{n}\right)$,

and is specified via its number of inputs $n$, its characteristic set 
International Journal of Mathematical, Engineering and Management Sciences

Vol. 4, No. 2, 306-326, 2019

https://dx.doi.org/10.33889/IJMEMS.2019.4.2-025

$\boldsymbol{A}=\left\{a_{0}, a_{1}, \ldots, a_{m}\right\} \subseteq \boldsymbol{I}_{n+1}=\{0,1,2, \ldots, n\},\{m \leq n\}$,

and its inputs $\boldsymbol{X}=\left[X_{1}, X_{2}, \ldots, X_{n}\right]^{\mathrm{T}}$. This function has the value 1 if and only if

$\sum_{i=1}^{n} X_{i}=a_{i}$

for all integers $i$ such that $0 \leq i \leq m$, and has the value 0 , otherwise. The $\operatorname{SSF} f$ in (1) can be alternatively expressed in terms of the complemented arguments $\bar{X}=\left[\overline{\mathrm{X}}_{1}, \overline{\mathrm{X}}_{2}, \ldots, \overline{\mathrm{X}}_{n}\right]^{\mathrm{T}}$ for an elementwise-complemented characteristic set given by

$\boldsymbol{E}=\left\{n-a_{m}, n-a_{m-1}, \ldots, n-a_{1}, n-a_{0}\right\}$

That means that the $S S F f$ in (1) can be also written as

$f=S y(n ; \boldsymbol{E} ; \overline{\boldsymbol{X}})=\operatorname{Sy}\left(n ;\left\{n-a_{m}, n-a_{m-1}, \ldots, n-a_{0}\right\} ; \overline{\mathrm{X}}_{1}, \overline{\mathrm{X}}_{2}, \ldots, \overline{\mathrm{X}}_{n}\right)$.

The complement $\bar{f}$ of the above $S S F$ has a characteristic set defined by the complementary set w.r.t. $\boldsymbol{I}_{n+1}$, given by the set difference $\left(\boldsymbol{I}_{n+1} / \boldsymbol{A}\right)$, which might be written as $\left(\boldsymbol{I}_{n+1}-\boldsymbol{A}\right)$ or

$\overline{\boldsymbol{A}}=\{0,1,2, \ldots, n\}-\left\{a_{0}, a_{1}, \ldots, a_{m}\right\}$,

and hence, $\bar{f}$ can be expressed as

$\bar{f}=S y(n ; \bar{A} ; \boldsymbol{X})$.

The ANDing and ORing of two SSFs of the same arguments $\boldsymbol{X}$, and of characteristic sets $\boldsymbol{A}_{\mathbf{1}}$ and $\boldsymbol{A}_{\mathbf{2}}$ are $S S F S$ with characteristic sets equal to the intersection and union of $\boldsymbol{A}_{\mathbf{1}}$ and $\boldsymbol{A}_{\mathbf{2}}$, respectively, i.e.,

$$
\begin{aligned}
& S y\left(n ; \boldsymbol{A}_{\mathbf{1}} ; \boldsymbol{X}\right) \wedge S y\left(n ; \boldsymbol{A}_{2} ; \boldsymbol{X}\right)=S y\left(n ; \boldsymbol{A}_{\mathbf{1}} \cap \boldsymbol{A}_{\mathbf{2}} ; \boldsymbol{X}\right) \\
& S y\left(n ; \boldsymbol{A}_{\mathbf{1}} ; \boldsymbol{X}\right) \quad \vee S y\left(n ; \boldsymbol{A}_{\mathbf{2}} ; \boldsymbol{X}\right)=S y\left(n ; \boldsymbol{A}_{\mathbf{1}} \cup \boldsymbol{A}_{\mathbf{2}} ; \boldsymbol{X}\right) .
\end{aligned}
$$

The Boole-Shannon expansion for the $S S F f$ in (1) about any of its variables $X_{m}(1 \leq m \leq n)$ can be stated as follows (Rushdi, 1986; 1993)

$S y(n ; \boldsymbol{A} ; \boldsymbol{X})=\bar{X}_{m} S y\left(n-1 ; \boldsymbol{B} ; \boldsymbol{X} / X_{m}\right) \vee X_{m} S y\left(n-1 ; \boldsymbol{C} ; \boldsymbol{X} / X_{m}\right), n \geq 0$,

where the two sets $\boldsymbol{B}$ and $\boldsymbol{C}$ are both subsets of the set $\boldsymbol{I}_{n}=\{0,1,2, \ldots, n-1\}$, as can be seen from their following definitions

$$
\begin{aligned}
& \boldsymbol{B}=\boldsymbol{A} \cap \boldsymbol{I}_{n}, \\
& \boldsymbol{D}=\left\{a_{0}-1, a_{1}-1, \ldots, a_{m}-1\right\}, \\
& \boldsymbol{C}=\boldsymbol{D} \cap \boldsymbol{I}_{n} .
\end{aligned}
$$


International Journal of Mathematical, Engineering and Management Sciences

Vol. 4, No. 2, 306-326, 2019

https://dx.doi.org/10.33889/IJMEMS.2019.4.2-025

The definitions of the two sets $\boldsymbol{B}$ and $\boldsymbol{C}$ might be restated as follows

$$
\begin{array}{ll}
\boldsymbol{B}=\boldsymbol{A} & \text { if } a_{m} \neq n, \\
\boldsymbol{B}=\boldsymbol{A}-\{n\} & \text { if } a_{m}=n, \\
\boldsymbol{C}=\boldsymbol{D} & \text { if } a_{0} \neq 0, \\
\boldsymbol{C}=\boldsymbol{D}-\{-1\} & \text { if } a_{0}=0 .
\end{array}
$$

The expansion (10) can be recursively applied till one of the following boundary conditions is reached

$\operatorname{Sy}\left(n ; \boldsymbol{I}_{n+1} ; \boldsymbol{X}\right)=1$

$\operatorname{Sy}(n ; \boldsymbol{\phi} ; \boldsymbol{X})=0$,

where $\boldsymbol{I}_{n+1}$ is the universe of discourse (universal set) for $n$ variables, and $\boldsymbol{\phi}=\{\}$ is the empty set (null set or set with no elements).

\section{Success of the binary k-out-of-n: G System}

The success $S(k, n, \boldsymbol{X})$ of a $k$-out-of- $n$ : G system is a monotonically non-decreasing symmetric $S S F$ given by (Rushdi, 1986; 1993)

$S(k, n, \boldsymbol{X})=\operatorname{Sy}(n ;\{m \mid k \leq m \leq n\} ; \boldsymbol{X})=\operatorname{Sy}(n ;\{k, k+1, \ldots, n\} ; \boldsymbol{X})$.

This success (like any coherent success) has the same minimal sum and complete sum. Each of these two sums is a disjunction of $\left(\begin{array}{l}n \\ k\end{array}\right)$ prime implicants, which are products of $k$ uncomplemented literals each. For example, the successes of $k$-out-of- 4 : G systems $(0 \leq k \leq 5)$ are

$$
\begin{aligned}
& S(0,4, \boldsymbol{X})=S y(4 ;\{0,1,2,3,4\} ; \boldsymbol{X})=1 \\
& S(1,4, \boldsymbol{X})=S y(4 ;\{1,2,3,4\} ; \boldsymbol{X})=X_{1} \vee X_{2} \vee X_{3} \vee X_{4}, \\
& S(2,4, \boldsymbol{X})=S y(4 ;\{2,3,4\} ; \boldsymbol{X})=X_{1} X_{2} \vee X_{1} X_{3} \vee X_{1} X_{4} \vee X_{2} X_{3} \vee X_{2} X_{4} \vee X_{3} X_{4},
\end{aligned}
$$

$S(3,4, \boldsymbol{X})=S y(4 ;\{3,4\} ; \boldsymbol{X})=X_{1} X_{2} X_{3} \vee X_{1} X_{2} X_{4} \vee X_{1} X_{3} X_{4} \vee X_{2} X_{3} X_{4}$,

$S(4,4, \boldsymbol{X})=S y(4 ;\{4\} ; \boldsymbol{X})=X_{1} X_{2} X_{3} X_{4}$

$S(5,4, \boldsymbol{X})=S y(4 ; \boldsymbol{\phi} ; \boldsymbol{X})=0$.

The six systems above are, respectively, the (fictitious) always successful system, the parallel (totally-redundant) system, the 2-out-of-4: $G$ (3-out-of-4: $F$ ) system, the fail-safe system, the (non-redundant) series system, and the (fictitious) always failed system (Rushdi, 1993; 2010). 
International Journal of Mathematical, Engineering and Management Sciences

Vol. 4, No. 2, 306-326, 2019

https://dx.doi.org/10.33889/IJMEMS.2019.4.2-025

The Boole-Shannon expansion for the success $S(k, n, \boldsymbol{X})$ of a $k$-out-of- $n$ : $\mathrm{G}$ system about any of its variables $X_{m}(1 \leq m \leq n)$ can be obtained as a restatement of equation (10) as the following recursive relation (Rushdi, 1993), which is valid for $(1 \leq k \leq n)$

$S(k, n, \boldsymbol{X})=\overline{\mathrm{X}}_{m} S\left(k, n-1, \boldsymbol{X} / X_{m}\right) \vee X_{m} S\left(k-1, n-1, \boldsymbol{X} / X_{m}\right)$.

This relation is used in conjunction with the following Boundary conditions, which simply restate the limiting cases (14) and (15)

$S(0, n, X)=1, n \geq 0$,

$S(n+1, n, X)=1, n \geq 0$,

Relations (18-20) can be represented by a compact regular "Signal Flow Graph" (SFG) that is essentially a variant of a "Reduced Ordered Binary Decision Diagram" (ROBDD) (Rushdi and Alturki, 2017; 2018a; 2018b). Based on this representation, iterative algorithms of quadratic temporal complexity and linear spatial complexity can be constructed for computing the reliability of various versions of $k$-out-of- $n$ systems (Rushdi, 1986; 1993; 2010; Rushdi et al., 2016).

The $k$-out-of- $n: G$ system is a coherent system such that

$S\left(k, n-1, X / X_{m}\right) \leq S\left(k-1, n-1, X / X_{m}\right)$,

and hence, (18) can be rewritten as

$S(k, n, \boldsymbol{X})=S\left(k, n-1, \boldsymbol{X} / X_{m}\right) \vee X_{m} S\left(k-1, n-1, \boldsymbol{X} / X_{m}\right)$.

Equation (22) means that $S(k, n, \boldsymbol{X})$ can be written as a formula of un-complemented variables only. In fact, this is possible for its minimal sum or complete sum, as exemplified by equations (17).

\section{Description of an Example Multi-State $\boldsymbol{k}$-out-of- $\boldsymbol{n}$ System}

While a binary $k$-out-of- $n$ : G system is a dichotomous system that is successful if and only if at least k out of its n components are successful, a multi-state $k$-out-of- $n$ : $G$ system is a multi-state system whose multi-valued success is greater than or equal to a certain value $j$ (lying between $l$ (the lowest output level) and $M$ (the highest output level)) whenever at least $k_{m}$ components are in state $m$ or above for all $m$ such that $1 \leq m \leq j$ (Tian et al., 2008; Mo et al., 2015).

In this section, we introduce a typical multi-state system that was discussed in Tian et al. (2008), Fadhel et al. (2014), and Mo et al. (2015). This is a commodity-supply system shown in Figure 1, in which a certain commodity (e.g., oil, water, energy, transportation traffic, or communication traffic) is delivered from the commodity source to three sinks (called stations) through four commodity pipelines. Both the system and each pipeline have four states, which are defined as follows. The states of each pipeline are defined according to which station(s) is/are reached by the commodity supply via this pipeline. Specifically. pipeline number $i$ is represented by a multivalued variable $X_{i}$, which has four values or instances $X_{i}\{j\},(1 \leq i \leq 4,0 \leq j \leq 3)$. The instance $X_{i}\{j\}$ is a binary indicator that the commodity can reach up to station $j$ through pipeline $i$. The four instances $X_{i}\{j\}$ are specified by the following propositions

$\left\{X_{i}\{0\}=1\right\} \Leftrightarrow\left\{X_{i}=0\right\} \Leftrightarrow$

\{Pipeline $i$ does not transmit the commodity to any station\}, 
International Journal of Mathematical, Engineering and Management Sciences

Vol. 4, No. 2, 306-326, 2019

https://dx.doi.org/10.33889/IJMEMS.2019.4.2-025

$\left\{X_{i}\{1\}=1\right\} \Leftrightarrow\left\{X_{i}=1\right\} \Leftrightarrow$

\{Pipeline $i$ transmits the commodity to station 1 only\},

$\left\{X_{i}\{2\}=1\right\} \quad \Leftrightarrow \quad\left\{X_{i}=2\right\} \quad \Leftrightarrow$

\{Pipeline $i$ transmits the commodity to stations 1 and 2 only\},

$\left\{X_{i}\{3\}=1\right\} \Leftrightarrow\left\{X_{i}=3\right\} \Leftrightarrow$

\{Pipeline $i$ transmits the commodity to stations 1,2 , and 3 \}.

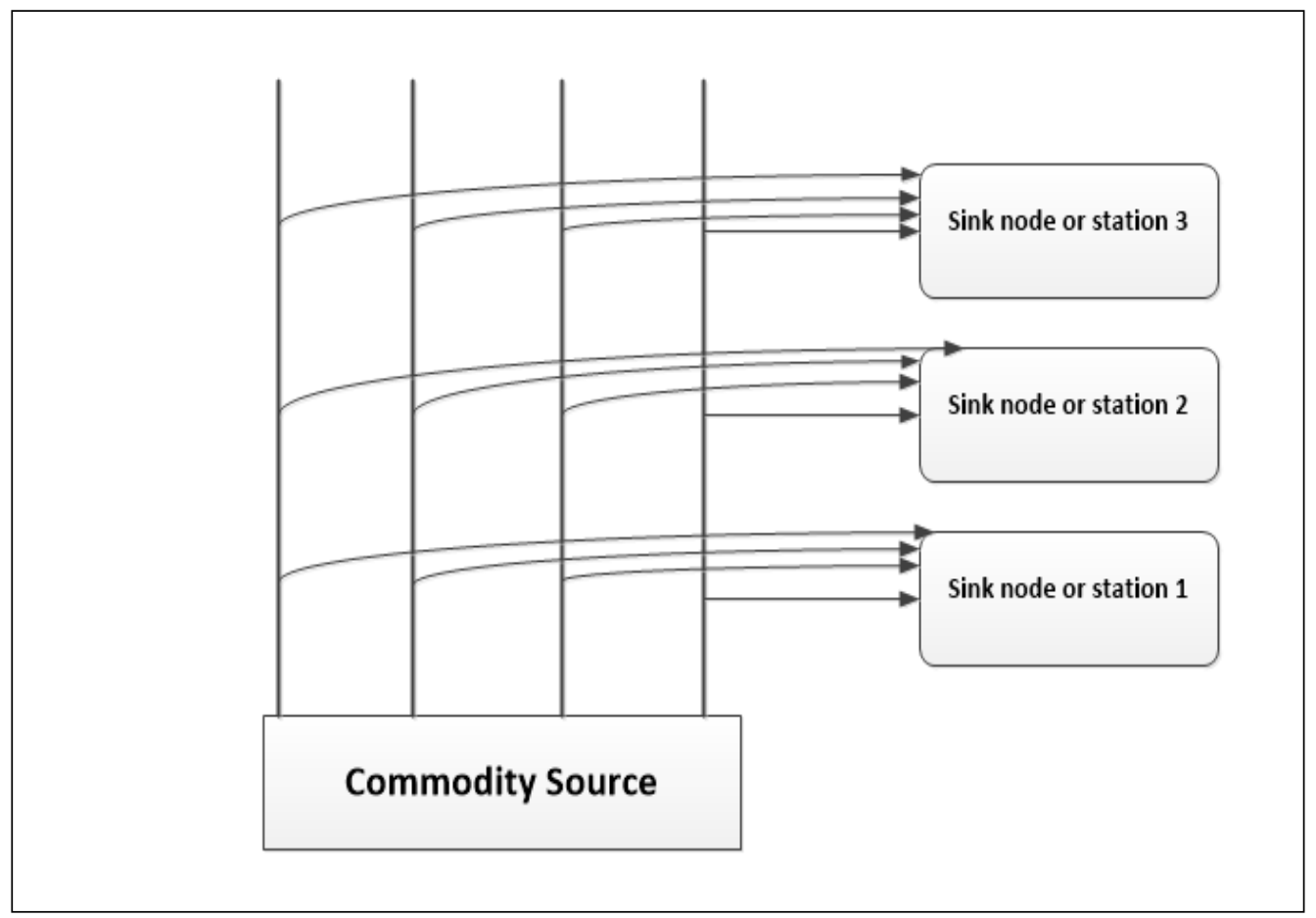

Figure 1. A commodity-supply system that is modeled as a multi-state k-out-of-n: G system (Adapted from Tian et al., 2008).

Note that a statement of the form $\left\{X_{i}=j\right\}$ assigns the value $j$ to the four-valued input variable $X_{i}$, while an equivalent statement of the form $\left\{X_{i}(j)=1\right\}$ asserts (sets to 1 ) its binary instance $X_{i}(j)$. While the four pipeline variables $X_{i}$ are statistically independent, the four instances for each of them are mutually exclusive and exhaustive. For $\{1 \leq i \leq 4\}$ and any permutation $\{j 0$, $j 1, j 2, j 3\}$ of the set $\{0,1,2,3\}$, each of these instances is related to the other ones via

$X_{i}\{j 0\}=\bar{X}_{i}\{j 1\} \wedge \bar{X}_{i}\{j 2\} \wedge \bar{X}_{i}\{j 3\}$ 
International Journal of Mathematical, Engineering and Management Sciences

Vol. 4, No. 2, 306-326, 2019

https://dx.doi.org/10.33889/IJMEMS.2019.4.2-025

$\bar{X}_{i}\{j 0\}=X_{i}\{j 1\} \vee X_{i}\{j 2\} \vee X_{i}\{j 3\}$

Equations (24) constitute a De-Morgan pair of relations, and mean that for $\{1 \leq i \leq 4\}$ the set of instances $\left\{X_{i}\{j\}, 0 \leq j \leq 3\right\}$ is an orthonormal set, i.e., in every possible configuration, one and only one of these four two-valued instances is 1 , while each of the remaining ones is 0 , namely

$X_{i}\{0\} \vee X_{i}\{1\} \vee X_{i}\{2\} \vee X_{i}\{3\}=1$,

$X_{i}\{j\} \wedge X_{i}\{k\}=0, \quad j \neq k$,

$X_{i}\{j\} \wedge \bar{X}_{i}\{k\}=X_{i}\{j\}, \quad j \neq k$.

In a similar fashion, the states of the system are defined according to whether the demands of up to a certain station can be met. We use $S\{k\}\{0 \leq k \leq 3\}$ to denote a binary indicator that the system can meet the commodity demand up to the station number $k$, i.e., for all stations $m(1 \leq$ $m \leq k)$. Note that station 0 does not exist, and hence $\{k=0\}$ means that the system cannot meet the commodity demand of any existing station. The four instances $S\{j\}$ are specified by the following propositions

$\{S\{0\}=1\} \Leftrightarrow\{S=0\} \Leftrightarrow$

\{The system does not meet the commodity demand of any station\},

$\{S\{1\}=1\} \Leftrightarrow\{S=1\} \Leftrightarrow$

\{The system meets the commodity demand of station 1 only\},

$\{S\{2\}=1\} \Leftrightarrow\{S=2\} \Leftrightarrow$

\{The system meets the commodity demand of stations 1 and 2 only\},

$\{S\{3\}=1\} \Leftrightarrow\{S=3\} \Leftrightarrow$

\{The system meets the commodity demand of stations 1,2 , and 3$\}$.

Note again that a statement of the form $\{S=k\}$ assigns the value $k$ to the four-valued output $S$, while a statement of the form $\{S(k)=1\}$ asserts (sets to 1 ) its binary instance $S(k)$. The four instances $S\{k\}, 0 \leq k \leq 3$ are mutually exclusive and exhaustive. For any permutation $\{j 0, j 1$, $j 2, j 3\}$ of the set $\{0,1,2,3\}$, each of these instances is related to the other ones via

$S\{j 0\}=\bar{S}\{j 1\} \wedge \bar{S}\{j 2\} \wedge \bar{S}\{j 3\}$,

$\bar{S}\{j 0\}=S\{j 1\} \vee S\{j 2\} \vee S\{j 3\}$.

Equations (27) are a De-Morgan pair of relations. They mean that the set of four instances $\{S\{k\}, 0 \leq k \leq 3\}$ is an orthonormal set, i.e., in every possible configuration, one and only one of these four two-valued instances is 1 , while each of the remaining ones is 0 , namely

$S\{0\} \vee S\{1\} \vee S\{2\} \vee S\{3\}=1$

$S\{j\} \wedge S\{k\}=0, \quad j \neq k$. 
International Journal of Mathematical, Engineering and Management Sciences

Vol. 4, No. 2, 306-326, 2019

https://dx.doi.org/10.33889/IJMEMS.2019.4.2-025

$S\{j\} \wedge \bar{S}\{k\}=S\{j\}, \quad j \neq k$.

Different stations have different demands on the commodity. Station 1 requires at least four pipelines working to meet its demand; Station 2 requires at least two pipelines working to meet its demand; Station 3 requires at least three pipelines working to meet its demand. Thus, this commodity-supply system can be regarded as a multi-state $k$-out-of- $n$ system with $n=4$, $M=3, k_{1}=4, k_{2}=2$, and $k_{3}=3$. Specifically, station 1 is an 4 -out-of-4: $G$ system with four inputs given by

$\bar{X}_{i}\{0\}=X_{i}\{1\} \vee X_{i}\{2\} \vee X_{i}\{3\}, \quad\{1 \leq i \leq 4\}$.

Station 2 is a $2-$ out-of $-4: G$ system with four inputs given by

$\bar{X}_{i}\{0\} \wedge \bar{X}_{i}\{1\}=X_{i}\{2\} \vee X_{i}\{3\}, \quad\{1 \leq i \leq 4\}$.

Finally, station 3 is a $3-$ out-of $-4: G$ system with four inputs given by

$\bar{X}_{i}\{0\} \wedge \bar{X}_{i}\{1\} \wedge \bar{X}_{i}\{2\}=X_{i}\{3\}, \quad\{1 \leq i \leq 4\}$.

We use $S_{m}\{1 \leq m \leq 3\}$ to depict the success of station $m$ (the indicator that the commodity demand of station $m$ is met). The successes of the three stations are given (in view of (17)) by

$$
\begin{aligned}
& S_{1}= S y\left(4 ;\{4\} ; \bar{X}_{1}\{0\}, \bar{X}_{2}\{0\}, \bar{X}_{3}\{0\}, \bar{X}_{4}\{0\}\right)=\bar{X}_{1}\{0\} \bar{X}_{2}\{0\} \bar{X}_{3}\{0\} \bar{X}_{4}\{0\} \\
& S_{2}= S y\left(4 ;\{2,3,4\} ; X_{1}\{2\} \vee X_{1}\{3\}, X_{2}\{2\} \vee X_{2}\{3\}, X_{3}\{2\} \vee X_{3}\{3\}, X_{4}\{2\} \vee X_{4}\{3\}\right) \\
&=\left(X_{1}\{2\} \vee X_{1}\{3\}\right)\left(X_{2}\{2\} \vee X_{2}\{3\}\right) \vee\left(X_{1}\{2\} \vee X_{1}\{3\}\right)\left(X_{3}\{2\} \vee X_{3}\{3\}\right) \\
& \vee\left(X_{1}\{2\} \vee X_{1}\{3\}\right)\left(X_{4}\{2\} \vee X_{4}\{3\}\right) \vee\left(X_{2}\{2\} \vee X_{2}\{3\}\right)\left(X_{3}\{2\} \vee X_{3}\{3\}\right) \\
& \vee\left(X_{2}\{2\} \vee X_{2}\{3\}\right)\left(X_{4}\{2\} \vee X_{4}\{3\}\right) \vee\left(X_{3}\{2\} \vee X_{3}\{3\}\right)\left(X_{4}\{2\} \vee X_{4}\{3\}\right),
\end{aligned}
$$

$$
\begin{aligned}
S_{3}= & S y\left(4 ;\{3,4\} ; X_{1}\{3\}, X_{2}\{3\}, X_{3}\{3\}, X_{4}\{3\}\right) \\
& =X_{1}\{3\} X_{2}\{3\} X_{3}\{3\} \vee X_{1}\{3\} X_{2}\{3\} X_{4}\{3\} \vee X_{1}\{3\} X_{3}\{3\} X_{4}\{3\} \vee X_{2}\{3\} X_{3}\{3\} X_{4}\{3\}
\end{aligned}
$$

\section{Analysis of the Example Multi-State System}

The four instances of the system output variable $S$ are related to station successes by

$$
\begin{aligned}
& S\{0\}=\overline{\mathrm{S}}_{1}, \\
& S\{1\}=S_{1} \overline{\mathrm{S}}_{2}, \\
& S\{2\}=S_{1} S_{2} \overline{\mathrm{S}}_{3}, \\
& S\{3\}=S_{1} S_{2} S_{3} .
\end{aligned}
$$


International Journal of Mathematical, Engineering and Management Sciences

Vol. 4, No. 2, 306-326, 2019

https://dx.doi.org/10.33889/IJMEMS.2019.4.2-025

The instances of $S$ can now be obtained by combining equations (30) and (31). In the following, we state the values of these instances and their expectations. First, we obtain $S\{0\}$ and its expectation (see Appendix A)

$S\{0\}=\overline{\mathrm{S}}_{1}=X_{1}\{0\} \vee X_{2}\{0\} \vee X_{3}\{0\} \vee X_{4}\{0\}$

$E\{S\{0\}\}=1-E\left\{\bar{X}_{1}\{0\}\right\} E\left\{\bar{X}_{2}\{0\}\right\} E\left\{\bar{X}_{3}\{0\}\right\} E\left\{\bar{X}_{4}\{0\}\right\}$.

Using properties (5) and (7) of SSFs, we complement the expression (30b) for $S_{2}$, we obtain

$$
\begin{aligned}
\bar{S}_{2}=S y\left(4 ;\{0,1\} ; X_{1}\{2\} \vee X_{1}\{3\}, X_{2}\{2\} \vee X_{2}\{3\}, X_{3}\{2\} \vee X_{3}\{3\}, X_{4}\{2\} \vee X_{4}\{3\}\right) \\
=S y\left(4 ;\{3,4\} ; X_{1}\{0\} \vee X_{1}\{1\}, X_{2}\{0\} \vee X_{2}\{1\}, X_{3}\{0\} \vee X_{3}\{1\}, X_{4}\{0\} \vee X_{4}\{1\}\right) .
\end{aligned}
$$

Using Eq. (B.3) of Boolean quotients, Eq. (17d) for $S y(4 ;\{3,4\} ; \boldsymbol{X})$ and Eq. (25c), we obtain

$$
\begin{aligned}
& S_{1} \overline{\mathrm{S}}_{2}=S_{1}\left(\overline{\mathrm{S}}_{2} / S_{1}\right) S\{1\}= \\
& \bar{X}_{1}\{0\} \bar{X}_{2}\{0\} \bar{X}_{3}\{0\} \bar{X}_{4}\{0\} \quad S y\left(4 ;\{3,4\} ; X_{1}\{1\}, X_{2}\{1\}, X_{3}\{1\}, X_{4}\{1\}\right)= \\
& =\bar{X}_{1}\{0\} \bar{X}_{2}\{0\} \bar{X}_{3}\{0\} \bar{X}_{4}\{0\}\left(X_{1}\{1\} X_{2}\{1\} X_{3}\{1\} \vee X_{1}\{1\} X_{2}\{1\} X_{4}\{1\} \vee X_{1}\{1\} X_{3}\{1\} X_{4}\{1\}\right. \\
& \left.\vee \quad X_{2}\{1\} X_{3}\{1\} X_{4}\{1\}\right) \\
& \quad=\quad X_{1}\{1\} X_{2}\{1\} X_{3}\{1\} \bar{X}_{4}\{0\} \vee X_{1}\{1\} X_{2}\{1\} \bar{X}_{3}\{0\} X_{4}\{1\} \vee X_{1}\{1\} \bar{X}_{2}\{0\} X_{3}\{1\} X_{4}\{1\} \vee \\
& \bar{X}_{1}\{0\} X_{2}\{1\} X_{3}\{1\} X_{4}\{1\} .
\end{aligned}
$$

The expression in Eq. (35) is not a $P R E$, but can be made so by utilizing Eq. (29a) as follows $S\{1\}=X_{1}\{1\} X_{2}\{1\} X_{3}\{1\}\left(X_{4}\{1\} \vee X_{4}\{2\} \vee X_{4}\{3\}\right) \vee$

$X_{1}\{1\} X_{2}\{1\}\left(X_{3}\{1\} \vee X_{3}\{2\} \vee X_{3}\{3\}\right) X_{4}\{1\} \vee$

$X_{1}\{1\}\left(X_{2}\{1\} \vee X_{2}\{2\} \vee X_{2}\{3\}\right) X_{3}\{1\} X_{4}\{1\} \vee$

$$
\left(X_{1}\{1\} \vee X_{1}\{2\} \vee X_{1}\{3\}\right) X_{2}\{1\} X_{3}\{1\} X_{4}\{1\} \text {. }
$$

Applying the distributive law to the $R H S$ of Eq. (36), we reduce it to 12 terms. One of these terms $\left(X_{1}\{1\} X_{2}\{1\} X_{3}\{1\} X_{4}\{1\}\right)$ appears four times and should be reduced (by virtue of idempotency) to one term, so as to obtain the following $P R E$, which consists of 9 disjoint terms

$S\{1\}=X_{1}\{1\} X_{2}\{1\} X_{3}\{1\}\left(X_{4}\{2\} \vee X_{4}\{3\}\right) \vee$

$X_{1}\{1\} X_{2}\{1\}\left(X_{3}\{2\} \vee X_{3}\{3\}\right) X_{4}\{1\} \vee$

$X_{1}\{1\}\left(X_{2}\{2\} \vee X_{2}\{3\}\right) X_{3}\{1\} X_{4}\{1\} \vee$

$$
\left(X_{1}\{2\} \vee X_{1}\{3\}\right) X_{2}\{1\} X_{3}\{1\} X_{4}\{1\} \vee X_{1}\{1\} X_{2}\{1\} X_{3}\{1\} X_{4}\{1\} \text {. }
$$

The PRE expression (37) is now converted directly, on a one-to-one basis, to the probability domain as follows

$$
\begin{gathered}
E\{S\{1\}\}=E\left\{X_{1}\{1\}\right\} E\left\{X_{2}\{1\}\right\} E\left\{X_{3}\{1\}\right\} \quad\left(E\left\{X_{4}\{2\}\right\}+E\left\{X_{4}\{3\}\right\}\right)+ \\
E\left\{X_{1}\{1\}\right\} E\left\{X_{2}\{1\}\right\}\left(E\left\{X_{3}\{2\}\right\}+E\left\{X_{3}\{3\}\right\}\right) E\left\{X_{4}\{1\}\right\}+ \\
E\left\{X_{1}\{1\}\right\}\left(\mathrm{E}\left\{X_{2}\{2\}\right\}+E\left\{X_{2}\{3\}\right\}\right) E\left\{X_{3}\{1\}\right\} \quad E\left\{X_{4}\{1\}\right\}+ \\
\left(E\left\{X_{1}\{2\}\right\}+E\left\{X_{1}\{3\}\right\}\right) E\left\{X_{2}\{1\}\right\} E\left\{X_{3}\{1\}\right\} E\left\{X_{4}\{1\}\right\}+ \\
E\left\{X_{1}\{1\}\right\} E\left\{X_{2}\{1\}\right\} E\left\{X_{3}\{1\}\right\} E\left\{X_{4}\{1\}\right\}
\end{gathered}
$$

Now, we compute $S_{1} S_{2}$ using (30a), (30b), (24b) and (25c) as 
International Journal of Mathematical, Engineering and Management Sciences

Vol. 4, No. 2, 306-326, 2019

https://dx.doi.org/10.33889/IJMEMS.2019.4.2-025

$$
\begin{gathered}
S_{1} S_{2}=\bar{X}_{1}\{0\} \bar{X}_{2}\{0\} \bar{X}_{3}\{0\} \bar{X}_{4}\{0\} \quad\left(( X _ { 1 } \{ 2 \} \vee X _ { 1 } \{ 3 \} ) ( X _ { 2 } \{ 2 \} \vee X _ { 2 } \{ 3 \} ) \vee \left(X_{1}\{2\} \vee\right.\right. \\
\left.X_{1}\{3\}\right)\left(X_{3}\{2\} \vee X_{3}\{3\}\right) \vee\left(X_{1}\{2\} \vee X_{1}\{3\}\right)\left(X_{4}\{2\} \vee X_{4}\{3\}\right) \vee\left(X_{2}\{2\} \vee X_{2}\{3\}\right)\left(X_{3}\{2\} \vee\right. \\
\begin{aligned}
&\left.X_{3}\{3\}\right) \vee\left(X_{2}\{2\} \vee X_{2}\{3\}\right)\left(X_{4}\{2\} \vee X_{4}\{3\}\right) \vee \\
&\left.\left(X_{3}\{2\} \vee X_{3}\{3\}\right)\left(X_{4}\{2\} \vee X_{4}\{3\}\right)\right) \\
&=\left(X_{1}\{2\} \vee X_{1}\{3\}\right)\left(X_{2}\{2\} \vee X_{2}\{3\}\right) \bar{X}_{3}\{0\} \bar{X}_{4}\{0\} \vee \\
&\left(X_{1}\{2\} \vee X_{1}\{3\}\right) \bar{X}_{2}\{0\}\left(X_{3}\{2\} \vee X_{3}\{3\}\right) \bar{X}_{4}\{0\} \vee \\
&\left(X_{1}\{2\} \vee X_{1}\{3\}\right) \bar{X}_{2}\{0\} \bar{X}_{3}\{0\}\left(X_{4}\{2\} \vee X_{4}\{3\}\right) \vee \\
& \bar{X}_{1}\{0\}\left(X_{2}\{2\} \vee X_{2}\{3\}\right)\left(X_{3}\{2\} \vee X_{3}\{3\}\right) \bar{X}_{4}\{0\} \vee \\
& \bar{X}_{1}\{0\}\left(X_{2}\{2\} \vee X_{2}\{3\}\right) \bar{X}_{3}\{0\}\left(X_{4}\{2\} \vee X_{4}\{3\}\right) \vee \\
& \bar{X}_{1}\{0\} \bar{X}_{2}\{0\}\left(X_{3}\{2\} \vee X_{3}\{3\}\right)\left(X_{4}\{2\} \vee X_{4}\{3\}\right) .
\end{aligned}
\end{gathered}
$$

Next, we compute $S_{1} S_{3}$, again using (30a), (30c), (24b) and (25c), as

$$
\begin{aligned}
& S_{1} S_{3}=\bar{X}_{1}\{0\} \bar{X}_{2}\{0\} \bar{X}_{3}\{0\} \bar{X}_{4}\{0\} \quad\left(X_{1}\{3\} X_{2}\{3\} X_{3}\{3\} \vee X_{1}\{3\} X_{2}\{3\} X_{4}\{3\} \vee\right. \\
& \left.X_{1}\{3\} X_{3}\{3\} X_{4}\{3\} \vee X_{2}\{3\} X_{3}\{3\} X_{4}\{3\}\right) \\
& =X_{1}\{3\} X_{2}\{3\} X_{3}\{3\} \bar{X}_{4}\{0\} \vee X_{1}\{3\} X_{2}\{3\} \bar{X}_{3}\{0\} X_{4}\{3\} \vee X_{1}\{3\} \bar{X}_{2}\{0\} X_{3}\{3\} X_{4}\{3\} \\
& \vee \bar{X}_{1}\{0\} X_{2}\{3\} X_{3}\{3\} X_{4}\{3\} \\
& =X_{1}\{3\} X_{2}\{3\} X_{3}\{3\}\left(X_{4}\{1\} \vee X_{4}\{2\} \vee X_{4}\{3\}\right) \\
& \vee X_{1}\{3\} X_{2}\{3\}\left(X_{3}\{1\} \vee X_{3}\{2\} \vee X_{3}\{3\}\right) X_{4}\{3\} \\
& \vee X_{1}\{3\}\left(X_{2}\{1\} \vee X_{2}\{2\} \vee X_{2}\{3\}\right) X_{3}\{3\} X_{4}\{3\} \\
& \vee\left(X_{1}\{1\} \vee X_{1}\{2\} \vee X_{1}\{3\}\right) X_{2}\{3\} X_{3}\{3\} X_{4}\{3\} \text {. }
\end{aligned}
$$

Applying the distributive law to the RHS of (40), we reduce it to 12 terms. One of these terms $\left(X_{1}\{3\} X_{2}\{3\} X_{3}\{3\} X_{4}\{3\}\right)$ appears four times and should be reduced (by virtue of idempotency) to one term, thereby resulting in the following $P R E$

$$
\begin{aligned}
S_{1} S_{3}= & X_{1}\{3\} X_{2}\{3\} X_{3}\{3\}\left(X_{4}\{2\} \vee X_{4}\{1\}\right) \\
& \vee X_{1}\{3\} X_{2}\{3\}\left(X_{3}\{2\} \vee X_{3}\{1\}\right) X_{4}\{3\} \vee X_{1}\{3\}\left(X_{2}\{2\} \vee X_{2}\{1\}\right) X_{3}\{3\} X_{4}\{3\} \\
& \vee\left(X_{1}\{2\} \vee X_{1}\{1\}\right) X_{2}\{3\} X_{3}\{3\} X_{4}\{3\} \vee X_{1}\{3\} X_{2}\{3\} X_{3}\{3\} X_{4}\{3\} .
\end{aligned}
$$

Comparing (39) to (41), and taking note of (29a), we observe that

$S_{1} S_{3} \leq S_{1} S_{2}$

which is a useful result, since it simplifies our expression for $S\{3\}$ to

$S\{3\}=S_{1} S_{2} S_{3}=\left(S_{1} S_{2}\right)\left(S_{1} S_{3}\right)=S_{1} S_{3}$.

As a consequence, $S\{3\}$ is given by the $P R E$ expression (41), which is now converted directly, on a one-to-one basis, to the probability domain as follows

$$
\begin{gathered}
E\{S\{3\}\}=E\left\{X_{1}\{3\}\right\} \quad E\left\{X_{2}\{3\}\right\} E\left\{X_{3}\{3\}\right\} \quad\left(E\left\{X_{4}\{2\}\right\}+E\left\{X_{4}\{1\}\right\}\right)+ \\
E\left\{X_{1}\{3\}\right\} E\left\{X_{2}\{3\}\right\}\left(E\left\{X_{3}\{2\}\right\}+E\left\{X_{3}\{1\}\right\}\right) E\left\{X_{4}\{3\}\right\}+ \\
E\left\{X_{1}\{3\}\right\}\left(E\left\{X_{2}\{2\}\right\}+E\left\{X_{2}\{1\}\right\}\right) E\left\{X_{3}\{3\}\right\} \quad E\left\{X_{4}\{3\}\right\}+ \\
\left(E\left\{X_{1}\{2\}\right\}+E\left\{X_{1}\{1\}\right\}\right) E\left\{X_{2}\{3\}\right\} E\left\{X_{3}\{3\}\right\} E\left\{X_{4}\{3\}\right\}+ \\
E\left\{X_{1}\{3\}\right\} E\left\{X_{2}\{3\}\right\} E\left\{X_{3}\{3\}\right\} E\left\{X_{4}\{3\}\right\} .
\end{gathered}
$$


International Journal of Mathematical, Engineering and Management Sciences

Vol. 4, No. 2, 306-326, 2019

https://dx.doi.org/10.33889/IJMEMS.2019.4.2-025

The similarity between (38) and (44) is striking, indeed. In fact, each of these two equations might be obtained from the other simply by interchanging the instances 1 and 3 for both inputs and output.

Now, the instance $S\{2\}$ of the multi-state four-valued variable $S$ is (by virtue of (31c) and (B.3))

$S\{2\}=S_{1} S_{2} \overline{\mathrm{S}}_{3}=S_{1}\left(S_{2} \overline{\mathrm{S}}_{3} / S_{1}\right)=S_{1}\left(S_{2} / S_{1}\right)\left(\overline{\mathrm{S}}_{3} / S_{1}\right)$

where $\left(S_{2} / S_{1}\right)$ is obtained from (30a), (29b), (30b) and (B.1) as

$$
\begin{gathered}
S_{2} / S_{1}=\operatorname{Sy}\left(4 ;\{2,3,4\} ; \bar{X}_{1}\{0\} \bar{X}_{1}\{1\}, \bar{X}_{2}\{0\} \bar{X}_{2}\{1\}, \bar{X}_{3}\{0\} \bar{X}_{3}\{1\}, \bar{X}_{4}\{0\} \bar{X}_{4}\{1\}\right) \\
\qquad / \bar{X}_{1}\{0\} \bar{X}_{2}\{0\} \bar{X}_{3}\{0\} \bar{X}_{4}\{0\} \\
=\operatorname{Sy}\left(4 ;\{2,3,4\} ; \bar{X}_{1}\{1\}, \bar{X}_{2}\{1\}, \bar{X}_{3}\{1\}, \bar{X}_{4}\{1\}\right),
\end{gathered}
$$

whose $P R E$ form is given as follows (with disjointing literals highlighted in red)

$$
\begin{array}{r}
S_{2} / S_{1}=\bar{X}_{1}\{1\} \bar{X}_{2}\{1\} \vee \bar{X}_{1}\{1\} X_{2}\{1\} \bar{X}_{3}\{1\} \vee \bar{X}_{1}\{1\} X_{2}\{1\} X_{3}\{1\} \bar{X}_{4}\{1\} \vee X_{1}\{1\} \bar{X}_{2}\{1\} \bar{X}_{3}\{1\} \vee \\
X_{1}\{1\} \bar{X}_{2}\{1\} X_{3}\{1\} \bar{X}_{4}\{1\} \vee X_{1}\{1\} X_{2}\{1\} \bar{X}_{3}\{1\} \bar{X}_{4}\{1\} .
\end{array}
$$

Now, we complement $S_{3}$ using (30c), (5) and (24a) to obtain $\overline{\mathrm{S}}_{3}$ as

$$
\begin{gathered}
\overline{\mathrm{S}}_{3}=\operatorname{Sy}\left(4 ;\{0,1,2\} ; X_{1}\{3\}, X_{2}\{3\}, X_{3}\{3\}, X_{4}\{3\}\right) \\
=\operatorname{Sy}\left(4 ;\{0,1,2\} ; \bar{X}_{1}\{0\} \bar{X}_{1}\{1\} \bar{X}_{1}\{2\}, \bar{X}_{2}\{0\} \bar{X}_{2}\{1\} \bar{X}_{2}\{2\}, \bar{X}_{3}\{0\} \bar{X}_{3}\{1\} \bar{X}_{3}\{2\},\right. \\
\left.\bar{X}_{4}\{0\} \bar{X}_{4}\{1\} \bar{X}_{4}\{2\}\right)
\end{gathered}
$$

and hence its quotient with respect to $S_{1}$ is

$$
\begin{aligned}
& \bar{S}_{3} / S_{1}=S y\left(4 ;\{0,1,2\} ; \bar{X}_{1}\{1\} \bar{X}_{1}\{2\}, \bar{X}_{2}\{1\} \bar{X}_{2}\{2\}, \bar{X}_{3}\{1\} \bar{X}_{3}\{2\}, \bar{X}_{4}\{1\} \bar{X}_{4}\{2\}\right)= \\
& S y\left(4 ;\{2,3,4\} ; X_{1}\{1\} \vee X_{1}\{2\}, X_{2}\{1\} \vee X_{2}\{2\}, X_{3}\{1\} \vee X_{3}\{2\}, X_{4}\{1\} \vee X_{4}\{2\}\right),
\end{aligned}
$$

which is given by the following $P R E$ form (again with disjointing literals highlighted in red)

$\overline{\mathrm{S}}_{3} / S_{1}=\left(X_{1}\{1\} \vee X_{1}\{2\}\right)\left(X_{2}\{1\} \vee X_{2}\{2\}\right) \vee\left(X_{1}\{1\} \vee X_{1}\{2\}\right) \bar{X}_{2}\{1\} \bar{X}_{2}\{2\}\left(X_{3}\{1\} \vee\right.$

$\left.X_{3}\{2\}\right) \vee\left(X_{1}\{1\} \vee X_{1}\{2\}\right) \bar{X}_{2}\{1\} \bar{X}_{2}\{2\} \bar{X}_{3}\{1\} \bar{X}_{3}\{2\}\left(X_{4}\{1\} \vee X_{4}\{2\}\right) \vee \bar{X}_{1}\{1\} \bar{X}_{1}\{2\}\left(X_{2}\{1\} \vee\right.$

$\left.X_{2}\{2\}\right)\left(X_{3}\{1\} \vee X_{3}\{2\}\right) \vee \bar{X}_{1}\{1\} \bar{X}_{1}\{2\}\left(X_{2}\{1\} \vee X_{2}\{2\}\right) \bar{X}_{3}\{1\} \bar{X}_{3}\{2\}\left(X_{4}\{1\} \vee X_{4}\{2\}\right) \vee$

$\bar{X}_{1}\{1\} \bar{X}_{1}\{2\} \bar{X}_{2}\{1\} \bar{X}_{2}\{2\}\left(X_{3}\{1\} \vee X_{3}\{2\}\right)\left(X_{4}\{1\} \vee X_{4}\{2\}\right)$.

Now, we can obtain $S\{2\}$ in PRE form via (45) by logically multiplying (ANDing) the three PREs of $S_{1}$ in (30a), $\left(S_{2} / S_{1}\right)$ in (46), and $\left(\overline{\mathrm{S}}_{3} / S_{1}\right)$ in (47), and making use of (25c):

$S\{2\}=\bar{X}_{1}\{0\} \bar{X}_{2}\{0\} \bar{X}_{3}\{0\} \bar{X}_{4}\{0\}\left(\left(X_{1}\{2\} X_{2}\{2\} \vee X_{1}\{2\} \bar{X}_{2}\{1\} \bar{X}_{2}\{2\}\left(X_{3}\{1\} \vee X_{3}\{2\}\right) \vee\right.\right.$ $X_{1}\{2\} \bar{X}_{2}\{1\} \bar{X}_{2}\{2\} \bar{X}_{3}\{1\} \bar{X}_{3}\{2\}\left(X_{4}\{1\} \vee X_{4}\{2\}\right) \vee \bar{X}_{1}\{1\} \bar{X}_{1}\{2\} X_{2}\{2\}\left(X_{3}\{1\} \vee X_{3}\{2\}\right) \vee$ $\bar{X}_{1}\{1\} \bar{X}_{1}\{2\} X_{2}\{2\} \bar{X}_{3}\{1\} \bar{X}_{3}\{2\}\left(X_{4}\{1\} \vee X_{4}\{2\}\right) \vee \bar{X}_{1}\{1\} \bar{X}_{1}\{2\} \bar{X}_{2}\{1\} \bar{X}_{2}\{2\}\left(X_{3}\{1\} \vee\right.$ $\left.\left.X_{3}\{2\}\right)\left(X_{4}\{1\} \vee X_{4}\{2\}\right)\right) \vee\left(X_{1}\{2\} X_{2}\{1\} \bar{X}_{3}\{1\} \vee 0 \vee 0 \vee \bar{X}_{1}\{1\} \bar{X}_{1}\{2\} X_{2}\{1\} X_{3}\{2\} \vee\right.$ $\left.\bar{X}_{1}\{1\} \bar{X}_{1}\{2\} X_{2}\{1\} \bar{X}_{3}\{1\} \bar{X}_{3}\{2\}\left(X_{4}\{1\} \vee X_{4}\{2\}\right) \vee 0\right) \vee\left(X_{1}\{2\} X_{2}\{1\} X_{3}\{1\} \bar{X}_{4}\{1\} \vee 0 \vee\right.$ $\left.0 \vee \bar{X}_{1}\{1\} \bar{X}_{1}\{2\} X_{2}\{1\} X_{3}\{1\} \bar{X}_{4}\{1\} \vee 0 \vee 0\right) \vee\left(X_{1}\{1\} X_{2}\{2\} \bar{X}_{3}\{1\} \vee X_{1}\{1\}\right.$ 
International Journal of Mathematical, Engineering and Management Sciences

Vol. 4, No. 2, 306-326, 2019

https://dx.doi.org/10.33889/IJMEMS.2019.4.2-025

$\left.\bar{X}_{2}\{1\} \bar{X}_{2}\{2\} X_{3}\{2\} \vee X_{1}\{1\} \bar{X}_{2}\{1\} \bar{X}_{2}\{2\} \bar{X}_{3}\{1\} \bar{X}_{3}\{2\}\left(X_{4}\{1\} \vee X_{4}\{2\}\right) \vee 0 \vee 0 \vee 0\right) \vee$

$\left(X_{1}\{1\} X_{2}\{2\} X_{3}\{1\} \bar{X}_{4}\{1\} \vee X_{1}\{1\} \bar{X}_{2}\{1\} \bar{X}_{2}\{2\} X_{3}\{1\} \bar{X}_{4}\{1\} \vee 0 \vee 0 \vee 0 \vee 0\right) \vee$

$\left.\left(X_{1}\{1\} X_{2}\{1\} \bar{X}_{3}\{1\} \bar{X}_{4}\{1\} \vee 0 \vee 0 \vee 0 \vee 0 \vee 0\right)\right)$.

The expression above in (48) is not a $P R E$, since statistical independence among multiplied quantities is violated by pairs $\bar{X}_{i}\{1\} \bar{X}_{i}\{2\}$ of instances of the same variable (marked in red). Note that any of these pairs can be replaced by its quotient with respect to the corresponding 0 -instance $\bar{X}_{i}\{0\}$, because this instance is a multiple of the entire expression. We utilize the identity

$$
\begin{aligned}
\bar{X}_{i}\{0\}\left(\bar{X}_{i}\{1\} \bar{X}_{i}\{2\}\right)=\bar{X}_{i}\{0\}\left(\left(\bar{X}_{i}\{1\} \bar{X}_{i}\{2\}\right) / \bar{X}_{i}\{0\}\right) \\
=\bar{X}_{i}\{0\}\left(\left(X_{i}\{0\} \vee X_{i}\{3\}\right) / \bar{X}_{i}\{0\}\right)=\bar{X}_{i}\{0\}\left(X_{i}\{3\} / \bar{X}_{i}\{0\}\right)=\bar{X}_{i}\{0\} X_{i}\{3\},
\end{aligned}
$$

to replace each pair of the form $\bar{X}_{i}\{1\} \bar{X}_{i}\{2\}$ in (48) by $X_{i}\{3\}$, thereby obtaining the following expression (in which locations where the form $X_{i}\{3\}$ emerges are marked in red)

$S\{2\}=\bar{X}_{1}\{0\} \bar{X}_{2}\{0\} \bar{X}_{3}\{0\} \bar{X}_{4}\{0\} \quad\left(X_{1}\{2\} X_{2}\{2\} \vee X_{1}\{2\} X_{2}\{3\}\left(X_{3}\{1\} \vee X_{3}\{2\}\right) \vee X_{1}\{2\}\right.$

$X_{2}\{3\} X_{3}\{3\}\left(X_{4}\{1\} \vee X_{4}\{2\}\right) \vee X_{1}\{3\} X_{2}\{2\}\left(X_{3}\{1\} \vee X_{3}\{2\}\right) \vee$

$X_{1}\{3\} X_{2}\{2\} X_{3}\{3\}\left(X_{4}\{1\} \vee X_{4}\{2\}\right) \vee X_{1}\{3\} X_{2}\{3\} \quad\left(X_{3}\{1\} \vee X_{3}\{2\}\right)\left(X_{4}\{1\} \vee X_{4}\{2\}\right) \vee$

$X_{1}\{2\} X_{2}\{1\} \bar{X}_{3}\{1\} \vee X_{1}\{3\} X_{2}\{1\} X_{3}\{2\} \vee X_{1}\{3\} X_{2}\{1\} X_{3}\{3\}\left(X_{4}\{1\} \vee X_{4}\{2\}\right) \vee$

$X_{1}\{2\} X_{2}\{1\} X_{3}\{1\} \bar{X}_{4}\{1\} \vee X_{1}\{3\} X_{2}\{1\} X_{3}\{1\} \bar{X}_{4}\{1\} \vee X_{1}\{1\} X_{2}\{2\} \bar{X}_{3}\{1\} \vee X_{1}\{1\}$

$X_{2}\{3\} X_{3}\{2\} \vee X_{1}\{1\} X_{2}\{3\} X_{3}\{3\}\left(X_{4}\{1\} \vee X_{4}\{2\}\right) \vee X_{1}\{1\} X_{2}\{2\} X_{3}\{1\} \bar{X}_{4}\{1\} \vee X_{1}\{1\}$

$\left.X_{2}\{3\} X_{3}\{1\} \bar{X}_{4}\{1\} \vee X_{1}\{1\} X_{2}\{1\} \bar{X}_{3}\{1\} \bar{X}_{4}\{1\}\right)$.

Expression (50) is not yet a PRE. We still need to multiply the common factor by the rest of the expression, where this factor gets partially or totally absorbed. Complemented instances of the form $\bar{X}_{i}\{1\}$ are also replaced by $\left(X_{i}\{2\} \vee X_{i}\{3\}\right)$ upon multiplication by $\bar{X}_{i}\{0\}$. The following expression for $S\{2\}$ is a $P R E$ (where changes in (50) are identified in red). The corresponding expectation $E\{S\{2\}\}$ is obvious, and will not be reproduced here for sake of brevity.

$$
\begin{aligned}
& S\{2\}=X_{1}\{2\} X_{2}\{2\} \bar{X}_{3}\{0\} \bar{X}_{4}\{0\} \vee X_{1}\{2\} X_{2}\{3\}\left(X_{3}\{1\} \vee X_{3}\{2\}\right) \bar{X}_{4}\{0\} \vee X_{1}\{2\} \\
& X_{2}\{3\} X_{3}\{3\}\left(X_{4}\{1\} \vee X_{4}\{2\}\right) \vee X_{1}\{3\} X_{2}\{2\}\left(X_{3}\{1\} \vee X_{3}\{2\}\right) \bar{X}_{4}\{0\} \vee \\
& X_{1}\{3\} X_{2}\{2\} X_{3}\{3\}\left(X_{4}\{1\} \vee X_{4}\{2\}\right) \vee X_{1}\{3\} X_{2}\{3\}\left(X_{3}\{1\} \vee X_{3}\{2\}\right)\left(X_{4}\{1\} \vee X_{4}\{2\}\right) \vee \\
& X_{1}\{2\} X_{2}\{1\}\left(X_{3}\{2\} \vee X_{3}\{3\}\right) \bar{X}_{4}\{0\} \vee X_{1}\{3\} X_{2}\{1\} X_{3}\{2\} \bar{X}_{4}\{0\} \vee \\
& X_{1}\{3\} X_{2}\{1\} X_{3}\{3\}\left(X_{4}\{1\} \vee X_{4}\{2\}\right) \vee X_{1}\{2\} X_{2}\{1\} X_{3}\{1\}\left(X_{4}\{2\} \vee X_{4}\{3\}\right) \vee \\
& X_{1}\{3\} X_{2}\{1\} X_{3}\{1\}\left(X_{4}\{2\} \vee X_{4}\{3\}\right) \vee X_{1}\{1\} X_{2}\{2\}\left(X_{3}\{2\} \vee X_{3}\{3\}\right) \bar{X}_{4}\{0\} \vee X_{1}\{1\} \\
& X_{2}\{3\} X_{3}\{2\} \bar{X}_{4}\{0\} \vee X_{1}\{1\} X_{2}\{3\} X_{3}\{3\}\left(X_{4}\{1\} \vee X_{4}\{2\}\right) \vee X_{1}\{1\} X_{2}\{2\} X_{3}\{1\}\left(X_{4}\{2\} \vee\right. \\
& \left.X_{4}\{3\}\right) \vee \vee X_{1}\{1\} X_{2}\{3\} X_{3}\{1\}\left(X_{4}\{2\} \vee X_{4}\{3\}\right) \vee X_{1}\{1\} X_{2}\{1\}\left(X_{3}\{2\} \vee X_{3}\{3\}\right)\left(X_{4}\{2\} \vee\right. \\
& \left.X_{4}\{3\}\right) .
\end{aligned}
$$

\section{Hand-Checking of Symbolic MSS Expressions}

The tedious computations in the preceding section produced lengthy symbolic formulas. Naturally, one might be skeptic concerning the susceptibility of these computations (and their outcomes) to numerical errors and/or fallacious reasoning. While there are methods for checking symbolic reliability expressions in the binary case (Rushdi, 1983), there seems to be none in the $M S S$ case. In the following, we present what we consider a first cut at the problem of checking a 
International Journal of Mathematical, Engineering and Management Sciences

Vol. 4, No. 2, 306-326, 2019

https://dx.doi.org/10.33889/IJMEMS.2019.4.2-025

symbolic MSS reliability expression. We present two kinds of checks herein. In the next section, we present an alternative method of verification through numerical comparison of results obtained by the present formulas to results obtained by other algorithms.

Our first check, which is simple and quick, utilizes an important characteristic of a binary function called its weight, which, when normalized, is called the syndrome or first spectral coefficient of the function. This is the number of true input vectors of the function, the number of truth-table lines or Karnaugh-map cells in which the function is asserted, or simply the number of minterms (Rushdi and Ghaleb, 2014). We check that the sum of the weights of the four instances $\{S\{k\}, 0 \leq k \leq 3\}$ is equal to the total number of minterms or $4^{4}=256$, namely

weight $(S\{0\})+$ weight $(S\{1\})+$ weight $(S\{2\})+$ weight $(S\{3\})=256$.

The availability of a $P R E$ for a binary function allows immediate computation of its weight through replacing logical multiplication and addition by their arithmetic counterparts, and replacing each uncomplemented instance of an input by 1 . In view of (27b), a complemented instance of an input is equal to the ORing of three disjoint uncomplemented instances, and hence should be replaced by 3 . Now, we use the PREs in (A.3), (37), (41), and (51) to obtain

$$
\begin{aligned}
& \text { weight }(\overline{\mathrm{S}}\{0\})=\text { weight }\left(\bar{X}_{1}\{0\} \bar{X}_{2}\{0\} \bar{X}_{3}\{0\} \bar{X}_{4}\{0\}\right)=3 * * 4=81, \\
& \text { weight }(S\{0\})=256-81=175, \\
& \text { weight }(S\{1\})=\operatorname{weight}(S\{3\})=9, \\
& \text { weight }(S\{2\})=63,
\end{aligned}
$$

which means that (52) is satisfied, as required.

A second check is more profound and more assuring (albeit more time consuming). It pertains to verifying identities (28), which attest that the set of instances $\{S\{k\}, 0 \leq k \leq 3\}$ is an orthonormal set. This can be accomplished by constructing the function table of the four-valued variable $S$ as a function of the four four-valued arguments $X_{1}, X_{2}, X_{3}$, and $X_{4}$. The input domain of this table has $4^{4}=256$ lines. A convenient form of this table is a Multi-Valued Karnaugh Map (MVKM) (Rushdi, 2018; Rushdi and Rushdi, 2018), whose map variables are the four fourvalued input variables $X_{i}\{1 \leq i \leq 4\}$, so that the map becomes as large as a conventional map of eight binary variables (Rushdi et al., 2019). An even more convenient form for the map is a variable-entered version of the MVKM (Rushdi, 2018). Figure 2 shows one such variable-entered MVKM using $X_{1}$ and $X_{3}$ as map variables, and $X_{2}$ and $X_{4}$ as entered variables. Figures 2(a)-(d) represent the four instances of $S$, and Figure 2(e) shows that the disjunction of these four instances of $S$ is identically equal to 1, while Figure 2(f) shows that the conjunction of any distinct two of these four instances of $S$ is identically equal to 0 . 
International Journal of Mathematical, Engineering and Management Sciences

Vol. 4, No. 2, 306-326, 2019

https://dx.doi.org/10.33889/IJMEMS.2019.4.2-025

\begin{tabular}{|c|c|c|c|c|}
\cline { 2 - 5 } \multicolumn{1}{c|}{} & $X_{1}\{0\}$ & $X_{1}\{1\}$ & $X_{1}\{3\}$ & $X_{1}\{2\}$ \\
\hline$X_{3}\{0\}$ & 1 & 1 & 1 & 1 \\
\hline$X_{3}\{1\}$ & 1 & $X_{2}\{0\} \vee X_{4}\{0\}$ & $X_{2}\{0\} \vee X_{4}\{0\}$ & $X_{2}\{0\} \vee X_{4}\{0\}$ \\
\hline$X_{3}\{3\}$ & 1 & $X_{2}\{0\} \vee X_{4}\{0\}$ & $X_{2}\{0\} \vee X_{4}\{0\}$ & $X_{2}\{0\} \vee X_{4}\{0\}$ \\
\hline$X_{3}\{2\}$ & 1 & $X_{2}\{0\} \vee X_{4}\{0\}$ & $X_{2}\{0\} \vee X_{4}\{0\}$ & $X_{2}\{0\} \vee X_{4}\{0\}$ \\
\hline
\end{tabular}

\begin{tabular}{|c|c|c|c|c|}
\cline { 2 - 5 } \multicolumn{1}{c|}{} & $X_{1}\{0\}$ & $X_{1}\{1\}$ & $X_{1}\{3\}$ & $X_{1}\{2\}$ \\
\hline$X_{3}\{0\}$ & 0 & 0 & 0 & 0 \\
\hline$X_{3}\{1\}$ & 0 & $\begin{array}{c}X_{2}\{1\}\left(X_{4}\{2\} \vee X_{4}\{3\}\right) \\
\vee\left(X_{2}\{2\} \vee X_{2}\{3\}\right) X_{4}\{1\} \\
\vee X_{2}\{1\} X_{4}\{1\}\end{array}$ & $X_{2}\{1\} X_{4}\{1\}$ & $X_{2}\{1\} X_{4}\{1\}$ \\
\hline$X_{3}\{3\}$ & 0 & $X_{2}\{1\} X_{4}\{1\}$ & 0 & 0 \\
\hline$X_{3}\{2\}$ & 0 & $X_{2}\{1\} X_{4}\{1\}$ & 0 & 0 \\
\hline
\end{tabular}

(b) $\boldsymbol{S}\{\mathbf{1}\}$

\begin{tabular}{|c|c|c|c|c|}
\cline { 2 - 6 } \multicolumn{1}{c|}{} & $X_{1}\{0\}$ & $X_{1}\{1\}$ & $X_{1}\{3\}$ & $X_{1}\{2\}$ \\
\hline$X_{3}\{0\}$ & 0 & 0 & 0 & 0 \\
\hline & 0 & 0 & $X_{2}\{3\} X_{4}\{3\}$ & 0 \\
$X_{3}\{1\}$ & 0 & $X_{2}\{3\}\left(X_{4}\{2\} \vee X_{4}\{1\}\right)$ & $X_{2}\{3\} X_{4}\{3\}$ \\
& & $X_{2}\{3\} X_{4}\{3\}$ & $\begin{array}{c}\left(X_{2}\{2\} \vee X_{2}\{1\}\right) X_{4}\{3\} \\
\vee X_{2}\{3\} X_{4}\{3\}\end{array}$ & 0 \\
$X_{3}\{3\}$ & 0 & $X_{2}\{3\} X_{4}\{3\}$ & 0 \\
\hline$X_{3}\{2\}$ & 0 & 0 & (c) $S\{3\}$ & \\
\hline
\end{tabular}

(c) $S\{3\}$

\begin{tabular}{|c|c|c|c|c|}
\hline & $X_{1}\{0\}$ & $X_{1}\{1\}$ & $X_{1}\{3\}$ & $X_{1}\{2\}$ \\
\hline$X_{3}\{0\}$ & 0 & 0 & 0 & 0 \\
\hline$X_{3}\{1\}$ & 0 & $\begin{array}{c}X_{2}\{2\}\left(X_{4}\{2\} \vee X_{4}\{3\}\right) \vee \\
X_{2}\{3\}\left(X_{4}\{2\} \vee X_{4}\{3\}\right)\end{array}$ & $\begin{array}{c}X_{2}\{2\} \bar{X}_{4}\{0\} \vee \\
X_{2}\{3\}\left(X_{4}\{1\} \vee X_{4}\{2\}\right) \vee \\
X_{2}\{1\}\left(X_{4}\{2\} \vee X_{4}\{3\}\right)\end{array}$ & $\begin{array}{c}\boldsymbol{X}_{2}\{2\} \overline{\boldsymbol{X}}_{4}\{0\} \vee \\
\boldsymbol{X}_{2}\{3\} \bar{X}_{4}\{0\} \vee \\
\boldsymbol{X}_{2}\{1\}\left(\boldsymbol{X}_{4}\{2\} \vee \boldsymbol{X}_{4}\{3\}\right)\end{array}$ \\
\hline$X_{3}\{3\}$ & 0 & $\begin{array}{c}X_{2}\{2\} \bar{X}_{4}\{0\} \vee \\
X_{2}\{3\}\left(X_{4}\{1\} \vee X_{4}\{2\}\right) \vee \\
X_{2}\{1\}\left(X_{4}\{2\} \vee X_{4}\{3\}\right)\end{array}$ & $\begin{array}{c}X_{2}\{2\}\left(X_{4}\{1\} \vee X_{4}\{2\}\right) \vee \\
X_{2}\{1\}\left(X_{4}\{1\} \vee X_{4}\{2\}\right)\end{array}$ & $\begin{array}{c}X_{2}\{2\} \bar{X}_{4}\{0\} \vee \\
X_{2}\{3\}\left(X_{4}\{1\} \vee X_{4}\{2\}\right) \vee \\
X_{2}\{1\} \bar{X}_{4}\{0\}\end{array}$ \\
\hline$X_{3}\{2\}$ & 0 & $\begin{array}{c}\boldsymbol{X}_{2}\{2\} \bar{X}_{4}\{0\} \vee \\
\boldsymbol{X}_{2}\{3\} \bar{X}_{4}\{0\} \vee \\
\boldsymbol{X}_{2}\{1\}\left(\boldsymbol{X}_{4}\{2\} \vee \boldsymbol{X}_{4}\{3\}\right)\end{array}$ & $\begin{array}{c}X_{2}\{2\} \bar{X}_{4}\{0\} \vee \\
X_{2}\{3\}\left(X_{4}\{1\} \vee X_{4}\{2\}\right) \vee \\
X_{2}\{1\} \bar{X}_{4}\{0\}\end{array}$ & $\begin{array}{c}X_{2}\{2\} \bar{X}_{4}\{0\} \vee \\
X_{2}\{3\} \bar{X}_{4}\{0\} \vee \\
X_{2}\{1\} \bar{X}_{4}\{0\}\end{array}$ \\
\hline
\end{tabular}

(d) $\boldsymbol{S}\{2\}$

\begin{tabular}{|c|c|c|c|c|}
\cline { 2 - 5 } \multicolumn{1}{c|}{} & $X_{1}\{0\}$ & $X_{1}\{1\}$ & $X_{1}\{3\}$ & $X_{1}\{2\}$ \\
\hline$X_{3}\{0\}$ & 1 & 1 & 1 & 1 \\
\hline$X_{3}\{1\}$ & 1 & 1 & 1 & 1 \\
\hline$X_{3}\{3\}$ & 1 & 1 & 1 & 1 \\
\hline$X_{3}\{2\}$ & 1 & 1 & 1 & 1 \\
\hline
\end{tabular}

\begin{tabular}{|c|c|c|c|c|}
\cline { 2 - 5 } \multicolumn{1}{c|}{} & $X_{1}\{\mathbf{0}\}$ & $X_{1}\{1\}$ & $X_{1}\{3\}$ & $X_{1}\{2\}$ \\
\hline$X_{3}\{0\}$ & 0 & 0 & 0 & 0 \\
\hline$X_{3}\{1\}$ & 0 & 0 & 0 & 0 \\
\hline$X_{3}\{3\}$ & 0 & 0 & 0 & 0 \\
\hline$X_{3}\{2\}$ & 0 & 0 & 0 & 0 \\
\hline
\end{tabular}

Figure 2. Variable-entered Karnaugh maps representing the four instances of the four-valued output variable and verifying their orthonormality 
International Journal of Mathematical, Engineering and Management Sciences

Vol. 4, No. 2, 306-326, 2019

https://dx.doi.org/10.33889/IJMEMS.2019.4.2-025

\section{Comparison with Previous Work}

The problem handled herein was solved via various multi-state techniques by Tian et al. (2008) and Mo et al. (2015). In both cases, the following input matrix was used, in which the sum of entries in each row is 1 (according to the expectation of (25a)).

$$
\left\{E\left\{X_{i}\{j\}\right\}\right\}=\left[\begin{array}{llll}
.050 & .0950 & .0684 & .7866 \\
.050 & .0950 & .0684 & .7866 \\
.030 & .0776 & .0446 & .8478 \\
.030 & .0776 & .0446 & .8478
\end{array}\right] \quad(1 \leq i \leq 4,0 \leq j \leq 3)
$$

Table 1 compares our results for this specific input with the results of the earlier two teams of authors. The three sets of results are essentially the same, despite the existence of minor differences in precision. For all the three sets of results, the sum of expectations of the four instances of the multi-valued output $S$ add exactly to one (within the precision used).

Table 1. Comparison of the present results with those in earlier work

\begin{tabular}{|c|c|c|c|}
\cline { 2 - 4 } \multicolumn{1}{c|}{} & Tian et al. (2008) & Mo et al. (2015) & Present Results \\
\hline $\mathbf{E}\{\mathbf{S}(\mathbf{0})\}$ & $\mathbf{0 . 1 5 0 8}$ & $\mathbf{0 . 1 5 0 8 3 8}$ & $\mathbf{0 . 1 5 0 8 3 7 7 5 0 0 0 0}$ \\
\hline $\mathbf{E}\{\mathbf{S}(\mathbf{1})\}$ & $\mathbf{0 . 0 0 2 3}$ & $\mathbf{0 . 0 0 2 2 8 2}$ & $\mathbf{0 . 0 0 2 2 8 2 5 4 8 1 2 8}$ \\
\hline $\mathbf{E}\{\mathbf{S}(\mathbf{2})\}$ & $\mathbf{0 . 0 8 9 2}$ & $\mathbf{0 . 0 8 9 1 8 1}$ & $\mathbf{0 . 0 8 9 1 8 0 8 6 6 4 3 6}$ \\
\hline $\mathbf{E}\{\mathbf{S}(\mathbf{3})\}$ & $\mathbf{0 . 7 5 7 7}$ & $\mathbf{0 . 7 5 7 6 9 9}$ & $\mathbf{0 . 7 5 7 6 9 8 8 3 5 4 3 6}$ \\
\hline Total & $\mathbf{1 . 0 0 0 0}$ & $\mathbf{1 . 0 0 0 0 0 0}$ & $\mathbf{1 . 0 0 0 0 0 0 0 0 0 0 0 0}$ \\
\hline
\end{tabular}

\section{Conclusions}

This paper demonstrated how MSS reliability can be handled via multi-valued logical tools. The techniques employed were purely algebraic, as opposed to ones employing the Karnaugh map (the map was used in a verification role only). The multi-valued inputs were used directly, as opposed to encoding them as equivalent binary variables. A classical MSS problem was manually analyzed. Results obtained satisfy important checks and replicate exactly earlier numerical results obtained by automated means.

\section{Appendix A: Probability-Ready Expressions}

The concept of a probability-ready expression $(R R E)$ is well-known in the two-valued logical domain (Rushdi and Rushdi, 2017), and it is still valid for the multi-valued logical domain. A ProbabilityReady Expression is a random expression that can be directly transformed, on a one-to-one basis, to its statistical expectation (its probability of being equal to 1 ) by replacing all logic variables by their statistical expectations and replacing disjunction and conjunction operations (ORing and ANDing) by their arithmetic counterparts of addition and multiplication. A logic expression is a PRE if

a) all disjuncted (ORed) terms (products) are mutually exclusive (disjoint), and

b) all conjuncted (ANDed) alterms (sums) are statistically independent. 
International Journal of Mathematical, Engineering and Management Sciences

Vol. 4, No. 2, 306-326, 2019

https://dx.doi.org/10.33889/IJMEMS.2019.4.2-025

Condition (a) is satisfied if for every pair of ORed terms, one term contains a certain instance of one of the multi-valued variables, while the other term contains another instance of the same variable. Condition (b) is satisfied if for every pair of ANDed alterms, one alterm involves variables describing a certain set of components, while the other alterm incorporates variables describing a mutually disjoint set (i.e., one of different components), under the assumption of independence of components. For example, the following expression

$S\{0\}=X_{1}\{0\} \vee X_{2}\{0\} \vee X_{3}\{0\} \vee X_{4}\{0\}$

is not a PRE, since it has ORed quantities that are not disjoint. A PRE version of it might be obtained by using a disjointing procedure (Rushdi and Rushdi, 2017), namely

$S\{0\}=X_{1}\{0\} \vee \bar{X}_{1}\{0\}\left(X_{2}\{0\} \vee \bar{X}_{2}\{0\}\left(X_{3}\{0\} \vee \bar{X}_{3}\{0\} X_{4}\{0\}\right)\right)$.

However, a much simpler PRE is obtained by simply complementing (A.1), namely

$$
\overline{\mathrm{S}}\{0\}=\bar{X}_{1}\{0\} \bar{X}_{2}\{0\} \bar{X}_{3}\{0\} \bar{X}_{4}\{0\} .
$$

The expression in (A.3) is a PRE since it has no ORed quantities while ANDed quantities in it are statistically independent. It results in the following expression, from which (11) is derived

$$
E\{\overline{\mathrm{S}}\{0\}\}=E\left\{\bar{X}_{1}\{0\}\right\} E\left\{\bar{X}_{2}\{0\}\right\} E\left\{\bar{X}_{3}\{0\}\right\} E\left\{\bar{X}_{4}\{0\}\right\}
$$

\section{Appendix B: Boolean Quotients}

The concept of a Boolean quotient is another switching-algebraic concept that can be conveniently viewed in a multi-valued context. Given a Boolean function $f$ and a product (term) $t$, the Boolean quotient of $f$ with respect to $t$, denoted by $(f / t)$ or $(f \mid t)$, is defined to be the function formed from $f$ by imposing the constraint $\{t=1\}$ explicitly (Brown, 1990), i.e.,

$$
f / t=[f]_{t=1},
$$

The Boolean quotient is also known as a ratio, a subfunction, or a restriction. Brown (1990) and Rushdi and Rushdi (2017) list several useful properties of Boolean quotients. A fundamental property of the Boolean quotient states that a term ANDed with a function is equal to the term ANDed with the Boolean quotient of the function with respect to the term, namely.

$$
t \wedge f=t \wedge(f / t)
$$

If the term $t$ is a factor of the function $f$ (i.e., $f=t \wedge g$, or $t \wedge f=f$ ) then (B.2) takes the simpler form (frequently utilized in this paper)

$$
f=t \wedge(f / t)
$$

The Boolean quotient can be denoted by an inclined slash $(f / t)$, or by a vertical bar $(f \mid t)$. The latter form is particularly insightful when associated with expectations. In fact, $E\{f \mid t\}$ appropriately expresses the conditional expectation of $f$ conditioned by $t$ or $f$ given $t$ (Rushdi and Al-Qwasmi, 2016; Rushdi and Rushdi, 2017). 
International Journal of Mathematical, Engineering and Management Sciences

Vol. 4, No. 2, 306-326, 2019

https://dx.doi.org/10.33889/IJMEMS.2019.4.2-025

\section{Appendix C: The Boole-Shannon Expansion}

The most effective way for converting a Boolean formula into a PRE form is the Boole-Shannon Expansion, which takes the following form in the two-valued case (Brown, 1990; Rushdi and Rushdi, 2017)

$$
f(\boldsymbol{X})=\left(\bar{X}_{i} \wedge f\left(\boldsymbol{X} \mid 0_{\mathrm{i}}\right)\right) \vee\left(X_{\mathrm{i}} \wedge f\left(\mathbf{X} \mid 1_{\mathrm{i}}\right)\right),
$$

This Boole-Shannon Expansion expresses a (two-valued) Boolean function $f(\boldsymbol{X})$ in terms of its two subfunctions $f\left(\boldsymbol{X} \mid 0_{\mathrm{i}}\right)$ and $f\left(\boldsymbol{X} \mid 1_{\mathrm{i}}\right)$. These subfunctions are equal to the Boolean quotients $f(\boldsymbol{X}) / \bar{X}_{i}$ and $f(\boldsymbol{X}) / X_{\mathrm{i}}$, and hence are obtained by restricting $\mathrm{X}_{i}$ in the expression $f(\boldsymbol{X})$ to 0 and 1 , respectively. If $f(\boldsymbol{X})$ is a sum-of-products expression of $n$ variables, the two subfunctions $f\left(\boldsymbol{X} \mid 0_{\mathrm{i}}\right)$ and $f\left(X \mid 1_{\mathrm{i}}\right)$ are functions of at most $(n-1)$ variables. A multi-valued extension of (C.1) in the context of this paper's example is

$$
\begin{aligned}
& S(\boldsymbol{X})=X_{i}\{0\} \wedge\left(S(\boldsymbol{X}) / X_{i}\{0\}\right) \vee X_{i}\{1\} \wedge\left(S(\boldsymbol{X}) / X_{i}\{1\}\right) \vee X_{i}\{2\} \wedge\left(S(\boldsymbol{X}) / X_{i}\{2\}\right) \vee \\
& X_{i}\{3\} \wedge\left(S(\boldsymbol{X}) / X_{i}\{3\}\right) .
\end{aligned}
$$

A formal proof of (C.2) is achieved by "perfect induction," that is, by considering four exhaustive distinct cases, namely: $\left\{X_{i}\{0\}=1\right\},\left\{X_{i}\{1\}=1\right\},\left\{X_{i}\{2\}=1\right\}$, and $\left\{X_{i}\{3\}=1\right\}$. In the first case, for example, $\left\{X_{i}\{0\}=1\right\}$, and consequently $\left\{X_{i}\{1\}=X_{i}\{2\}=X_{i}\{3\}=0\right\}$. Therefore,

The LHS of $(\mathrm{C} .2)=$ the RHS of $(\mathrm{C} .2)=S(\boldsymbol{X}) \mid\left(X_{i}\{0\}=1\right)=S(\boldsymbol{X}) / X_{i}\{0\}$.

The other three cases can be handled in a similar fashion. The expansion (C.2) serves our purposes very well. Once the subfunctions in (C.2) are expressed by $P R E$ expressions, $S(\mathbf{X})$ will be also in $P R E$ form, thanks to the combined two facts:

(a) The RHS of (C.2) has four disjoint terms, each of which containing one of the four disjoint instances $X_{i}\{0\}, X_{i}\{1\}, X_{i}\{2\}$, and $X_{i}\{3\}$ of the variable $X_{i}$,

(b) Each of these four terms is a product of two statistically-independent entities, since any subfunction $S(\boldsymbol{X}) / X_{i}\{j\}(0 \leq j \leq 3)$ does not involve any instance of the four-valued variable $X_{i}$.

The expansion (C.2) transforms to the probability domain as

$$
\begin{aligned}
& E\{S(\boldsymbol{X})\}=E\left\{X_{i}\{0\}\right\} * E\left\{S(\boldsymbol{X}) / X_{i}\{0\}\right\}+E\left\{X_{i}\{1\}\right\} * E\left\{S(\boldsymbol{X}) / X_{i}\{1\}\right\}+E\left\{X_{i}\{2\}\right\} * \\
& E\left\{S(\boldsymbol{X}) / X_{i}\{2\}\right\} * E\left\{X_{i}\{3\}\right\} * E\left\{S(\boldsymbol{X}) / X_{i}\{3\}\right\} .
\end{aligned}
$$

Equation (C.4) is nothing but a restatement of the Total Probability Theorem, with our understanding that the expectation of a Boolean quotient is simply a conditional probability (Rushdi and AlQwasmi, 2016). It is the basis of multi-valued decision diagrams (MDDs) for the reliability analysis of multi-state systems (Mo, 2014; Mo et al., 2014; 2015). 
International Journal of Mathematical, Engineering and Management Sciences

Vol. 4, No. 2, 306-326, 2019

https://dx.doi.org/10.33889/IJMEMS.2019.4.2-025

\section{Conflict of Interest}

The author declares that no competing interests exist.

\section{Acknowledgement}

The author benefited from (and is grateful for) his earlier collaboration and enlightening discussions with Engineer Mahmoud Ali Rushdi, Research Scientist at fortiss (Forschungsinstitut des Freistaats Bayern für softwareintensive Systeme und Services ("Research Institute of the Free State of Bavaria for softwareintensive Systems and Services”)), Munich, Germany.

\section{References}

Amari, S. V., Xing, L., Shrestha, A., Akers, J., \& Trivedi, K. S. (2010). Performability analysis of multistate computing systems using multivalued decision diagrams. IEEE Transactions on Computers, 59(10), 1419-1433.

Arnold, R. F., \& Harrison, M. A. (1963). Algebraic properties of symmetric and partially symmetric Boolean functions. IEEE Transactions on Electronic Computers, EC-12(3), 244-251.

Born, R. C., \& Scidmore, A. K. (1968). Transformation of switching functions to completely symmetric switching functions. IEEE Transactions on Computers, C-17(6), 596-599.

Brown, F. M. (1990). Boolean Reasoning: The Logic of Boolean Equations, Kluwer Academic Publishers, Boston, MA, USA.

Caldwell, S. H. (1954). The recognition and identification of symmetric switching functions. Transactions of the American Institute of Electrical Engineers, Part I: Communication and Electronics, 73(2), 142147.

Canteaut, A., \& Videau, M. (2005). Symmetric Boolean functions. IEEE Transactions on Information Theory, 51(8), 2791-2811.

Cunkle, C. H. (1963). Symmetric Boolean functions. The American Mathematical Monthly, 70(8), 833-836.

Ding, Y., Zio, E., Li, Y., Cheng, L., \& Wu, Q. (2012). Definition of multi-state weighted k-out-of-n: F systems. International Journal of Performability Engineering, 8(2), 217-219.

Fadhel S. F., Alauldin N. A., \& Ahmed Y. Y. (2014). Reliability of dynamic multi-state oil supply system by structure function. International Journal of Innovative Research in Science, Engineering and Technology, 3(6), 13548-13555.

Hill, F. J., \& Peterson, G. R. (1993). Computer aided logical design with emphasis on VLSI. $4^{\text {th }}$ Edition, Wiley, New York, NY, USA.

Khorshidi, H. A., Gunawan, I., \& Ibrahim, M. Y. (2015). On reliability evaluation of multistate weighted kout-of-n system using present value. The Engineering Economist, 60(1), 22-39.

Kim, B. G., \& Dietmeyer, D. L. (1991). Multilevel logic synthesis of symmetric switching functions. IEEE Transactions on Computer-Aided Design of Integrated Circuits and Systems, 10(4), 436-446.

Kravets, V. N., \& Sakallah, K. A. (2000). Generalized symmetries in Boolean functions. In Proceedings of the 2000 IEEE/ACM International Conference on Computer-Aided Design (pp. 526-532). IEEE Press.

Lee, S. C. (1978). Modern switching theory and digital design, Prentice-Hall, Englewood Cliffs, New Jersey, NJ, USA.

Levitin, G. (2013). Multi-state vector- $k$-out-of-n systems. IEEE Transactions on Reliability, 62(3), 648657. 
International Journal of Mathematical, Engineering and Management Sciences

Vol. 4, No. 2, 306-326, 2019

https://dx.doi.org/10.33889/IJMEMS.2019.4.2-025

Levitin, G., Lisnianski, A., \& Ushakov, I. (2003). Reliability of multi-state systems: a historical overview. In Lindqvist, B. H. \& Doksum, K. A. (Editors), World Scientific, Mathematical and Statistical Methods in Reliability (pp. 123-137).

Li, C. Y., Chen, X., Yi, X. S., \& Tao, J. Y. (2011). Interval-valued reliability analysis of multi-state systems. IEEE Transactions on Reliability, 60(1), 323-330.

Li, S., Sun, S., Si, S., Zhang, S., \& Dui, H. (2014). Decision diagram based methods and reliability analysis for $k$-out-of- $n$ : G systems. Journal of Mechanical Science and Technology, 28(10), 3917-3923.

Li, Z., \& Kapur, K. C. (2011). Models and measures for fuzzy reliability and relationship to multi-state reliability. International Journal of Performability Engineering, 7(3), 241-250.

Marcus, M. P. (1956). The detection and identification of symmetric switching functions with the use of tables of combinations. IRE Transactions on Electronic Computers, EC-5(4), 237-239.

Maurer, P. M. (2015). Symmetric Boolean functions. International Journal of Mathematics, Game Theory, and Algebra, 24(2/3), 159-202.

Mishchenko, A. (2003). Fast computation of symmetries in Boolean functions. IEEE Transactions on Computer-Aided Design of Integrated Circuits and Systems, 22(11), 1588-1593.

Mo, Y. (2014). A multiple-valued decision-diagram-based approach to solve dynamic fault trees. IEEE Transactions on Reliability, 63(1), 81-93.

Mo, Y., Xing L., \& Amari, S. V. (2014). A multiple-valued decision diagram based method for efficient reliability analysis of non-repairable phased-mission systems. IEEE Transactions on Reliability, 63(1), 320-330.

Mo, Y., Xing, L., Amari, S. V., \& Dugan, J. B. (2015). Efficient analysis of multi-state $k$-out-of- $n$ systems. Reliability Engineering \& System Safety, 133, 95-105.

Muroga, S. (1979). Logic design and switching theory. John Wiley \& Sons, New York, NY, USA.

Muselli, M. (2005). Approximate multi-state reliability expressions using a new machine learning technique. Reliability Engineering \& System Safety, 89(3), 261-270.

Ram, M. (2013). On system reliability approaches: a brief survey. International Journal of System Assurance, Engineering, and Management, 4(2), 101-117.

Ren, Y., Zeng, C., Fan, D., Liu, L., \& Feng, Q. (2018). Multi-state reliability assessment method based on the MDD-GO model. IEEE Access, 6, 5151-5161.

Rushdi A. M. A. (2010). Partially-redundant systems: examples, reliability, and life expectancy. International Magazine on Advances in Computer Science and Telecommunications. 1(1), 1-13.

Rushdi M. A. M., Ba-Rukab O. M. \& Rushdi A. M. (2016). Multidimensional recursive relation and mathematical induction techniques: The case of failure frequency of $k$-out-of- $n$ systems. Journal of King Abdulaziz University: Engineering Science, 27(2), 15-31.

Rushdi, A. M. \& Rushdi, M. A. (2017). Switching-algebraic analysis of system reliability, Chapter 6 in M. Ram and P. Davim (Editors), Advances in Reliability and System Engineering. Cham, Switzerland: Springer International Publishing, pp. 139-161.

Rushdi, A. M. (1983). How to hand-check a symbolic reliability expression. IEEE Transactions on Reliability, $R$-32(5), 402-408.

Rushdi, A. M. (1986). Utilization of symmetric switching functions in the computation of $k$-out-of- $n$ system reliability. Microelectronics and Reliability, 26(5), 973-987. 
International Journal of Mathematical, Engineering and Management Sciences

Vol. 4, No. 2, 306-326, 2019

https://dx.doi.org/10.33889/IJMEMS.2019.4.2-025

Rushdi, A. M. (1993). Reliability of $k$-out-of- $n$ systems, Chapter 5 in Misra, K. B. (Editor), New Trends in System Reliability Evaluation, Vol. 16, Fundamental Studies in Engineering, Elsevier Science Publishers, Amsterdam, The Netherlands, pp. 185-227.

Rushdi, A. M. A. (2018). Utilization of Karnaugh maps in multi-value qualitative comparative analysis. International Journal of Mathematical, Engineering and Management Sciences, 3(1), 28-46.

Rushdi, A. M. A., \& Al-Qwasmi, M. A. (2016). Exposition and comparison of two kinds of a posteriori analysis of fault trees. Journal of King Abdulaziz University: Computing and Information Technology, 5(1), 55-74.

Rushdi, A. M. A., \& Alturki, A. M. (2017). Computation of $k$-out-of- $n$ system reliability via reduced ordered binary decision diagrams. British Journal of Mathematics \& Computer Science, 22(3), 1-9.

Rushdi, A. M. A., \& Alturki, A. M. (2018b). Unification of mathematical concepts and algorithms of $k$-outof- $n$ system reliability: A perspective of improved disjoint products. Journal of Engineering Research. $6(4), 1-31$.

Rushdi, A. M. A., \& Ghaleb, F. A. M. (2014). The Walsh spectrum and the real transform of a switching function: A review with a Karnaugh-map perspective. Journal of Engineering and Computer Sciences, Qassim University, 7, 73-112.

Rushdi, A. M., \& Alturki, A. M. (2018a). Novel representations for a coherent threshold reliability system: a tale of eight signal flow graphs. Turkish Journal of Electrical Engineering \& Computer Sciences, 26(1), 257-269.

Rushdi, A. M., Zagzoog, S., \& Balamesh, A. S. (2019). Derivation of a scalable solution for the problem of factoring an n-bit integer. Journal of Advances in Mathematics and Computer Science, 30(1), 1-22.

Rushdi, R. A., \& Rushdi, A. M. (2018). Karnaugh-map utility in medical studies: The case of Fetal Malnutrition. International Journal of Mathematical, Engineering and Management Sciences, 3(3), 220-244.

Shrestha, A., Xing, L., \& Dai, Y. (2007). MBDD versus MMDD for multistate systems analysis. In Dependable, Autonomic and Secure Computing, 2007. DASC 2007. Third IEEE International Symposium on (pp. 172-180). IEEE.

Shrestha, A., Xing, L., \& Dai, Y. (2010). Decision diagram-based methods and complexity analysis for multi-state systems. IEEE Transactions on Reliability, 59(1), 145-161.

Singh, V. V., \& Ram, M. (2014). Multi-state $k$-out-of- $n$ type system analysis. Mathematics in Engineering Science \& Aerospace, 5(3), 281-292.

Song, X., Zhai, Z., Guo, Y., Zhu, P., \& Han, J. (2017). Approximate analysis of multi-state weighted $k$-outof- $n$ systems applied to transmission lines. Energies, 10(11), 1740, doi:10.3390/en10111740.

Tian, Z., Zuo, M. J., \& Yam, R. C. (2008). Multi-state $k$-out-of- $n$ systems and their performance evaluation. IIE Transactions, 41(1), 32-44.

Unger, S. H. (1989). The essence of logic circuits. Englewood Cliffs, NJ, Prentice Hall, 1989, pp. 317.

Veeraraghavan, M., \& Trivedi, K. S. (1994). A combinatorial algorithm for performance and reliability analysis using multistate models. IEEE Transactions on Computers, 43(2), 229-234.

Zang, X., Wang, D., Sun, H., \& Trivedi, K. S. (2003). A BDD-based algorithm for analysis of multistate systems with multistate components. IEEE Transactions on Computers, 52(12), 1608-1618.

Zhao, X., \& Cui, L. (2010). Reliability evaluation of generalised multi-state $k$-out-of- $n$ systems based on FMCI approach. International Journal of Systems Science, 41(12), 1437-1443. 
International Journal of Mathematical, Engineering and Management Sciences

Vol. 4, No. 2, 306-326, 2019

https://dx.doi.org/10.33889/IJMEMS.2019.4.2-025

Zuo, M. J., Tian, Z., \& Huang, H. Z. (2007). An efficient method for reliability evaluation of multistate networks given all minimal path vectors. IIE Transactions, 39(8), 811-817.

(c) (i) Original content of this work is copyright $\odot$ International Journal of Mathematical, Engineering and Management Sciences. All rights reserved. Except of uses under a Creative Commons Attribution 4.0 International (CC BY 4.0) license at https://creativecommons.org/licenses/by/4.0/ 